\title{
Paracorporeal lung assist devices as a bridge to recovery or lung transplantation in neonates and young children
}

\author{
David M. Hoganson, MD, ${ }^{\mathrm{a}}$ Avihu Z. Gazit, MD, ${ }^{\mathrm{b}, \mathrm{c}}$ Umar S. Boston, MD, ${ }^{\mathrm{a}}$ Stuart C. Sweet, MD, ${ }^{\mathrm{d}}$ \\ R. Mark Grady, MD, ${ }^{c}$ Charles B. Huddleston, $\mathrm{MD},{ }^{\mathrm{e}}$ and Pirooz Eghtesady, MD, $\mathrm{PhD}^{\mathrm{a}}$
}

Objective: To evaluate paracorporeal lung assist devices to treat neonates and children with decompensated respiratory failure as a bridge to recovery or lung transplantation.

\begin{abstract}
Methods: One neonate (23 days old) and 3 young children (aged 2, 9, and 23 months) presented with primary lung disease with pulmonary hypertension, including alveolar capillary dysplasia in 2 and right pulmonary hypoplasia and primary pulmonary hypertension in 1 . The patients were listed for lung transplantation but decompensated and required extracorporeal membrane oxygenation (ECMO). The patients were transitioned from ECMO to a pumpless paracorporeal lung assist device (Maquet Quadrox-iD oxygenator in 3, Novalung in 1) with inflow from the pulmonary artery and return to the left atrium.
\end{abstract}

Results: The patients were weaned from ECMO and supported by the device for $44 \pm 29$ days (range, 5-74). Three patients were extubated while supported by the device (after 9,15 , and 72 days). One patient was bridged to lung transplant ( 9 months old, with alveolar capillary dysplasia, supported 5 days). One patient was bridged to recovery with maximal medical therapy ( 23 months old, with primary pulmonary hypertension, supported 23 days). Two patients died while awaiting a suitable lung donor after a support time of 54 and 72 days.

Conclusions: Pediatric patients bridged from ECMO to lung transplantation have poor results. An alternative method for longer term respiratory support was necessary as a bridge for these patients. The use of a paracorporeal lung assist device successfully supported 4 patients to recovery, lung transplantation, or past the average wait time for pediatric donor lungs (27 days). This therapy has the potential to bridge children with decompensated respiratory failure to lung transplantation. (J Thorac Cardiovasc Surg 2014;147:420-7)

Neonates or small children with end-stage lung disease who develop respiratory failure requiring extracorporeal membrane oxygenation (ECMO) have few clinical options. Some of these patients can be weaned from ECMO, especially if their condition can be managed with optimized medical therapy. This, however, is an uncommon scenario, and survival for these patients has been $<50 \%$ and has often been accompanied by significant complications. ${ }^{1}$ Although these children can be listed for lung transplantation (LT), the post-transplant results for patients receiving ECMO, particularly venoarterial, have been poor. ${ }^{2}$ Given these

From the Division of Cardiothoracic Surgery, ${ }^{\mathrm{a}}$ Department of Surgery, Division of Critical Care, ${ }^{\mathrm{b}}$ Division of Cardiology, ${ }^{\mathrm{c}}$ and Division of Allergy, Immunology, and Pulmonary Medicine, ${ }^{\mathrm{d}}$ Department of Pediatrics, St Louis Children's Hospital and Washington University; and Division of Pediatric Cardiothoracic Surgery, Department of Surgery, St Louis University, St Louis, Mo.

Disclosures: David M. Hoganson reports consulting fees and grant support from the Kensey Nash Corporation. The other authors have nothing to disclose with regard to commercial support.

Read at the 93rd Annual Meeting of The American Association for Thoracic Surgery, Minneapolis, Minnesota, May 4-8, 2013.

Received for publication April 14, 2013; revisions received Aug 16, 2013; accepted for publication Aug 27, 2013; available ahead of print Nov 6, 2013.

Address for reprints: Pirooz Eghtesady, MD, PhD, Section of Pediatric Cardiothoracic Surgery, Division of Cardiothoracic Surgery, Department of Surgery, Washington University School of Medicine, Campus Box 8234, $660 \mathrm{~S}$ Euclid Ave, St Louis, MO 63110 (E-mail: eghtesadyp@wudosis.wustl.edu).

$0022-5223 / \$ 36.00$

Copyright (c) 2014 by The American Association for Thoracic Surgery

http://dx.doi.org/10.1016/j.jtcvs.2013.08.078 poor outcomes, our philosophy has been to reconsider the candidacy of listed patients if their clinical deterioration has warranted ECMO support. This clinical conundrum led us to seek alternative options for support, as a bridge to lung transplantation or recovery, in listed patients with clinical decompensation.

Recently, adult patients with end-stage respiratory failure and pulmonary hypertension who have become decompensated and required ECMO have been successfully bridged to lung transplantation with the support of the Novalung oxygenator (Novalung GmbH, Heilbronn, Germany) using central cannulation with blood inflow from the pulmonary artery (PA) and blood return to the left atrium (LA). ${ }^{3-5}$ Many of these patients have been extubated and participated in physical therapy during support.

The present report summarizes our experience with the first series of infants and children with severe respiratory failure who were supported with a centrally placed paracorporeal pumpless oxygenator in a PA to LA configuration. Each of these patients had pulmonary hypertension and preserved right ventricular function. These prerequisites were intentionally chosen, because of the presumption that the elevated pulmonary vascular resistance would result in shunting blood flow through the lower resistance paracorporeal oxygenator. All patients were initially treated with an intent of a bridge to lung transplantation. 


$$
\begin{aligned}
& \text { Abbreviations and Acronyms } \\
& \begin{aligned}
\text { ACD } & =\text { alveolar capillary dysplasia } \\
\text { ATIII } & =\text { antithrombin III } \\
\text { AV } & =\text { atrioventricular } \\
\text { ECMO } & =\text { extracorporeal membrane oxygenation } \\
\text { LA } & =\text { left atrium } \\
\text { LT } & =\text { lung transplantation } \\
\text { PA } & =\text { pulmonary artery }
\end{aligned}
\end{aligned}
$$

\section{METHODS}

\section{Patient Presentation}

One neonate (aged 23 days) and 3 young children (aged 2, 9, and 23 months) presented with primary lung disease, including alveolar capillary dysplasia (ACD) in 2, horseshoe lung with right pulmonary hypoplasia, pulmonary interstitial glycogenosis, and an atrioventricular (AV) canal defect in 1, and primary pulmonary hypertension in 1. All these patients had pulmonary hypertension demonstrated by echocardiography. All patients had been evaluated and listed for LT at their clinical deterioration requiring venoarterial ECMO support despite maximal medical therapy. These patients were considered for paracorporeal oxygenator support with central cannulation. Each case was reviewed with the university human research protection office and our institutional multidisciplinary ethics team, and extensive and open discussions were had with each family, who agreed to proceed.

\section{Technique of Implanting Paracorporeal Oxygenator With Central Cannulation}

Each patient was in the intensive care unit receiving ECMO support with right carotid artery and right internal jugular venous cannulation before being brought to the operating room. Of the 4 patients, 3 were transitioned to traditional cardiopulmonary bypass by way of a median sternotomy. The 23-month-old patient was transitioned directly from ECMO to the paracorporeal Novalung support. For PA cannulation, an aortic Berlin Heart cannula (Berlin Heart AG, Berlin, Germany) was sewn to the main PA. The 6-mm version of the cannula was used in the 3 infants and the 9-mm version in the 23-month-old child. In 2 patients, an interposed short piece of 8-mm Gore-Tex shunt (W. L. Gore \& Associates, Flagstaff, Ariz) was used between the 6-mm cannula and the PA (with a pericardial washer on the Gore-Tex graft) to allow for easier implantation and to improve the hemostasis of the cannula to PA anastomosis. For the LA shunt, in the first 3 patients, a metal tip right angle cannula (DLP Medtronics Inc, Grand Rapids, Mich) was inserted into the LA through a pursestring suture $(16 \mathrm{~F}$ in the 23 day old neonate and 2-month-old infant and 22F in the 23-month-old child). After observations of significant thrombus formation around and inside the metal cannula tip from the first few cases, 1 of us (P.E.) devised an alternative approach for the LA cannulation (Figure 1). This technique was used in the fourth patient, a 9-month-old infant with ACD. LA cannulation was performed using a 6-mm Berlin arterial cannula modified with a 10-mm-diameter Gore-Tex graft extension. The 10-mm graft attached to the Berlin cannula was anastomosed to a surgically created atrial septal defect (corresponding to the area of a Blalock-Hanlon incision) by way of a right atriotomy. The right atrial incision was then closed around the $10-\mathrm{mm}$ shunt. In all patients, the cannulas were passed through the chest wall, and the oxygenator was positioned just below the patient's feet, supported by a custom holding device. The patient with the AV canal underwent complete repair of the $\mathrm{AV}$ canal during the same operation as the transition to paracorporeal oxygenator support.

For the 23-month-old child, who was the first to be treated, ${ }^{1}$ the Novalung oxygenator was used to support the patient. However, the minimum recommended flow of the Novalung $(500 \mathrm{~mL} / \mathrm{min})$ was greater than the expected blood flow through the device for the neonate and infants. Therefore, for the infants, the Quadrox iD oxygenator (Maquet, Wayne, $\mathrm{NJ}$ ), with a minimum recommended flow of $200 \mathrm{~mL} / \mathrm{min}$, was used. Similar to the Novalung, the Quadrox iD was designed to have a low gradient across the oxygenator. A low-flow air-oxygen mixer (Sechrist Industries, Inc, Anaheim, Calif) was used to control the sweep and inspired oxygen percentage in the oxygenator. Blood flow through the oxygenator was monitored continuously at the bedside using a Transonic HT110 flowmeter (Transonic Systems, Inc, Ithaca, NY). Pressures proximal and distal to the oxygenator were continuously monitored in the postoperative period.

\section{Anticoagulation Management of Paracorporeal Oxygenator}

Anticoagulation was achieved using a heparin infusion with a targeted activated clotting time of 160 to 200 seconds in 3 patients, consistent with what has been reported for adults who have been treated with the paracorporeal Novalung with central cannulation. ${ }^{5,6}$ In the neonatal patient, a target activated clotting time of 180 to 220 seconds was achieved, and aspirin was also given, as has been reported for adult Novalung patients. ${ }^{7}$ The neonate and 2 infants also had their antithrombin III (ATIII) levels monitored. ATIII was replaced with recombinate ATIII (Atryn, ABO Pharmaceuticals, San Diego, Calif) to maintain levels $>80 \%$. The circuit, including cannulas, tubing, connectors, and oxygenator, was monitored for thrombus buildup by visual inspection and continuous measurement of the transmembrane gradient and postmembrane blood gases. An increase in the transmembrane gradient of the oxygenator greater than the baseline suggested potential thrombus buildup in the oxygenator. If that occurred, the oxygenator and connectors were changed out at the bedside, with a circuit clamp times of $<1$ minute.

\section{RESULTS}

These 4 patients were supported by ECMO for a period of $8 \pm 7$ days. During the operation to transition to oxygenator support, adequate flow through the oxygenator allowed prompt weaning of cardiopulmonary bypass or ECMO (in the Novalung patient). The duration of oxygenator support was $44 \pm 29$ days (range, 5-74). The patients and support times on the paracorporeal oxygenator are listed in Table 1. One patient ( 9 months old, with ACD, supported for 5 days) was bridged to lung transplantation and was doing well 10 months after transplantation. One patient (23 months old) had primary pulmonary hypertension and was supported on the device while the pulmonary vasodilators were escalated. After 22 days of oxygenator support, the patient developed an embolic stroke and was taken to the operating room, where the Novalung oxygenator was successfully weaned. The patient has continued to receive an intravenous pulmonary vasodilator and was doing well at home 34 months after weaning from the paracorporeal oxygenator support, with minimal neurologic sequelae from his stroke. Two patients died while awaiting a suitable lung donor after a paracorporeal oxygenator support time of 54 and 74 days. The neonatal patient with ACD was supported for 54 days and was extubated after 15 days of support. ${ }^{8}$ However, that patient experienced a hemorrhagic stroke after 44 days of support that progressed during the next 10 days despite 


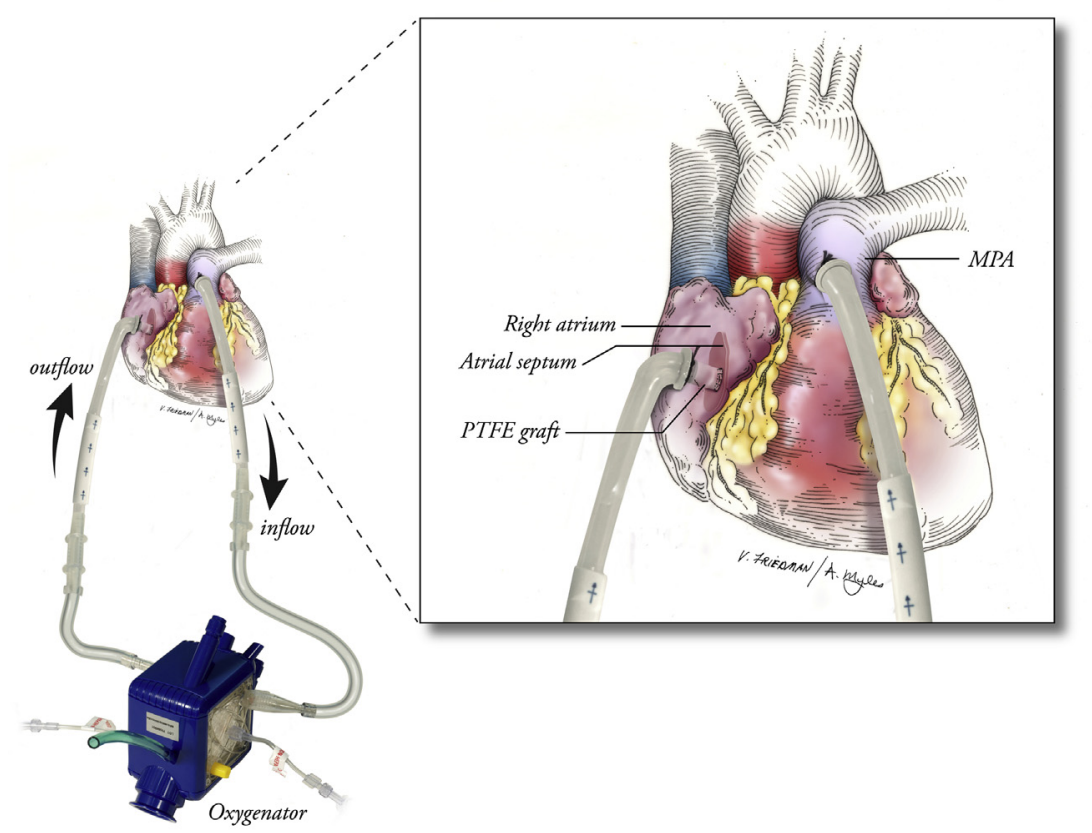

FIGURE 1. Diagram demonstrating the cannulation of the left atrium for the fourth patient treated with paracorporeal oxygenator support. A 10-mm Gore-Tex graft extension was added to a 6-mm Berlin cannula. The Gore-Tex graft was passed through the right atrium and sewn to the interatrial septum. $M P A$, Main pulmonary artery; PTFE, polytetrafluoroethylene.

intermittently holding anticoagulation. The patient was taken off the LT waiting list, transitioned to comfort care, and died. The 2-month-old infant with the AV canal defect was supported for 72 days and experienced a small hemorrhagic stroke after 17 days of support, but this did not progress. The patient's course was complicated by need to return to the operating room for treatment of a residual atrial septal defect, resulting in significant left to right shunting, in part from small left-sided structures and elevated left ventricular end-diastolic pressure that were appreciated subsequently. This patient eventually developed renal failure during oxygenator support. The patient was taken off the lung transplant waiting list, and the family decided to transition to comfort care. The patient was extubated on postoperative day 72 and died on postoperative day 74 .

All these patients had pulmonary hypertension with suprasystemic PA pressures (mean systolic pulmonary pressure, $98 \pm 21 \mathrm{~mm} \mathrm{Hg}$ ). With the low resistance of the oxygenator circuit and elevated pulmonary vascular resistance in these patients, the flow through the circuit was substantial in all patients. The resistance of the oxygenator circuit was lower than the pulmonary vascular resistance, as evidenced by the measured preoxygenator pressures during device support were lower than the preoperative right ventricle pressures (Table 2). We measured a pressure decrease across the Quadrox iD oxygenator, with a range from 6 to $14 \mathrm{~mm} \mathrm{Hg}$ at a flow of 324 to $884 \mathrm{~mL} / \mathrm{min}$. The flow and the mean preoxygenator pressure for the neonate and 9-month-old patient supported with the Quadrox are shown in Figure 2. Overall, the flow remained within a narrow range for most of the treatment duration.

The balance of blood flow through the oxygenator circuit and the native lungs cannot be directly measured. However, transthoracic echocardiography of the neonate during paracorporeal support demonstrated some flow reversal within the main PA, with substantial flow from the LA cannula and some residual flow in the right and left pulmonary veins. This suggested that most of the

TABLE 1. Overview of patient diagnoses, support times, and outcome

\begin{tabular}{lccccc}
\hline Age & Diagnosis & ECMO support (d) & Lung device support (d) & Extubated after device insertion (d) & Outcome \\
\hline $23 \mathrm{~d}$ & ACD & 5 & 54 & 15 & Death \\
$2 \mathrm{mo}$ & AV canal, R lung hypoplasia & 0.1 & 74 & 72 & Death \\
$9 \mathrm{mo}$ & ACD & 9 & 5 & NA & Lung transplant \\
$23 \mathrm{mo}$ & PPH & 17 & 42 & 9 & Weaned to medical \\
& & & & management \\
\hline
\end{tabular}

ECMO, Extracorporeal membrane oxygenation; $A C D$, alveolar capillary dysplasia; $A V$, arteriovenous; $R$, right; $P P H$, primary pulmonary hypertension. 
TABLE 2. Demonstration of right ventricular unloading by paracorporeal lung assist device as evidenced by a decrease in pulmonary artery pressures

\begin{tabular}{lcccc}
\hline Age & Diagnosis & Preoperative PASP* $(\mathbf{m m ~ H g})$ & Preoperative SBP $(\mathbf{m m}$ Hg) & PASP during device support $+(\mathbf{m m}$ Hg) \\
\hline $23 \mathrm{~d}$ & ACD & 75 & 50 & 55 \\
$2 \mathrm{mo}$ & AV canal, R lung hypoplasia & 125 & 85 & 51 \\
$9 \mathrm{mo}$ & ACD & 100 & 75 & 46 \\
$23 \mathrm{mo}$ & PPH & 93 & 75 & Not measured \\
\hline
\end{tabular}

$P A S P$, Pulmonary artery systolic pressure; $S B P$, systemic systolic blood pressure; $A C D$, alveolar capillary dysplasia; $A V$, arteriovenous; $R$, right; $P P H$, primary pulmonary hypertension. *As estimated using echocardiography. †Premembrane pressure plus measured cannula pressure decrease.

right-sided blood flow was going through the oxygenator circuit, with the minority of flow through the native lungs. This was further evidenced in that the total measured blood flow through the oxygenator was substantial given the weight of the children. For example, the $3.3-\mathrm{kg}$ neonate had an oxygenator flow of $500 \mathrm{~mL} / \mathrm{min}$.

The relative resistance of the different cannulation strategies used in the LA can be compared by assessing
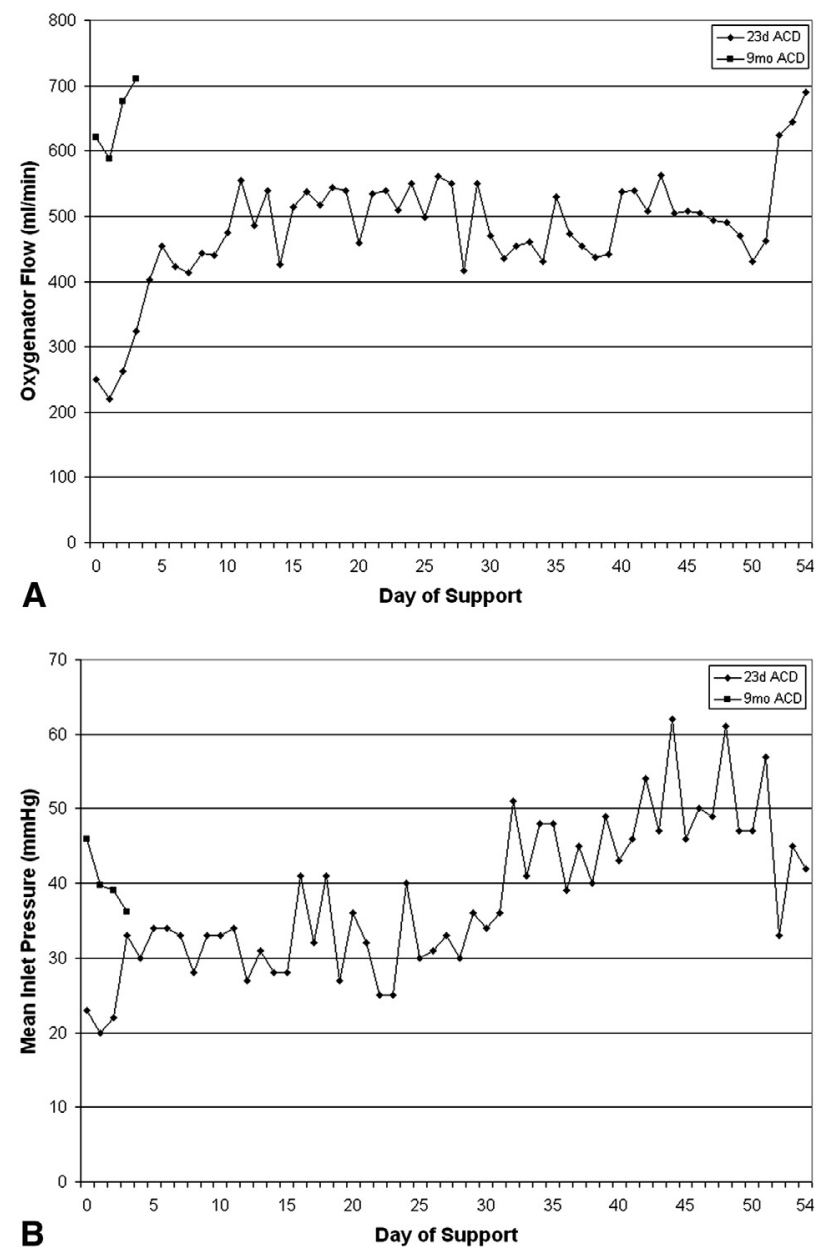

FIGURE 2. Comparison of hemodynamics of Quadrox iD oxygenator paracorporeal support of the 2 patients with alveolar capillary dysplasia treated with the device. A, Flow through the oxygenator. B, Mean oxygenator inlet pressures. the postoxygenator pressure. The postoxygenator pressure was the sum of the LA pressure and the pressure decrease across the cannula returning the blood to the LA. Using the $16 \mathrm{~F}$, right angle, metal cannula for LA return in the 23-day-old patient, the postoxygenator pressure generally ranged from 20 to $32 \mathrm{~mm} \mathrm{Hg}$, depending on the device flow. The conversion from a $16 \mathrm{~F}$, right angle, metal cannula to the 6-mm Berlin cannula with the 10-mm Gore-Tex shunt to the LA resulted in a significant decrease in the resistance of the outflow cannula, as evidenced by a $5-\mathrm{mm} \mathrm{Hg}$ lower postoxygenator pressure compared with that with the $16 \mathrm{~F}$ cannula in the 23-day-old patient when the periods of equal overall flow were compared.

\section{DISCUSSION}

Pediatric patients with end-stage respiratory disease who decompensate and require ECMO have limited options if they are unable to be weaned from ECMO within a period of weeks. The present case series has demonstrated the feasibility of a new treatment option for these patients. Using a paracorporeal oxygenator as a lung assist device, respiratory support was provided for these patients.

Ideally, this paracorporeal support should be lower risk than ECMO and provide adequate support for these patients for a period while they await LT. Furthermore, this approach clearly facilitates rehabilitation, including extubation, potential mobilization, and nutritional support of children while awaiting LT. This was not too dissimilar to the patients with heart failure who were rehabilitated with a paracorporeal ventricular assist device, with resulting positive outcomes after heart transplantation. With the average wait time for a pediatric LT recipient of 27 days (range, 2 to 121), ${ }^{9}$ all these patients were supported well past the average time to LT, with the exception of the patient who underwent LT after only 5 days of support. This duration of support was similar to the adult experience with Novalung and central cannulation, in which 9 patients underwent bridged to LT, with support times of 8 to 69 days. $^{3-5}$ Certainly, for some patients with decompensated pulmonary disease, weaning from the support might be possible, which was accomplished in 1 patient in our series.

In the 3 patients treated with the Quadrox oxygenator, their premembrane pressures were measured continuously postoperatively, allowing estimation of the right ventricular 
systolic pressure according to the intraoperative measured pressure decrease across the cannula from the PA to the oxygenator. These data have demonstrated a clear unloading of the right ventricle when the patients are receiving paracorporeal oxygenator support (Table 2). Our previous experience in pediatric patients with heart failure-related pulmonary hypertension treated with a biventricular assist device showed that decompression of the pulmonary vascular bed allows reverse remodeling of the pulmonary vasculature. ${ }^{1,10}$ One patient with primary pulmonary hypertension was weaned from the paracorporeal lung assist device in the present study. It could be postulated that right ventricular and pulmonary vasculature unloading by the paracorporeal oxygenator circuit contributed to the ability to wean the patient from the device because his pulmonary vasodilators were increased. For patients who proceed to LT, the unloading of the right ventricle will still be beneficial and will be optimized as cannulation strategies continue to evolve to create a lower resistance circuit.

The safety and comfort with the paracorporeal systems for ambulation and the extubated state might be greater than with ECMO, just as was noted for circulatory support. Two patients in our series were extubated for most of their time during support; in 1 patient, respiratory failure persisted, in part because of the less than optimal hemodynamics. Overall, our experience was in line with that observed in adults who received similar therapy and were able to be extubated and participate in physical therapy while awaiting LT. Our extubated pediatric patients were able to interact with their families in a meaningful way, take oral feedings, and participate in some physical activity. The latter also resulted in substantial reductions in the need for sedative and narcotic medications, which could have additional implications for clinical outcomes.

Application of this technology to these patients who are in extreme decompensation at surgery presents with significant challenges in making the therapy available while obtaining the right degree of approval and consent. In the present study, each case was treated individually, with review by the university human research protection office and our ethics team. The institutional review board approved our use of the Novalung. Additional application of this therapy should include prospective institutional review board approval, such that appropriate research data can be collected for a careful study of this approach.

Anticoagulation management of these oxygenators in infants was challenging because of the low flow rates and small cardiac structures. Added to that challenge was the metal, right angle cannula in the LA in 3 patients. The high risk of thromboembolism led us to apply an aggressive anticoagulation regimen, which increased the risk of hemorrhage. Their platelet dysfunction caused by treatment with medications with platelet inhibitory effects, such as milrinone, also increased their risk of hemorrhage. The ATIII levels were maintained at $>80 \%$; however, that was entirely empiric, because at that time we did not use thromboelastography monitoring. ATIII supplementation causing an increased heparin effect could place the patient at additional risk of bleeding. However, with more extensive monitoring, such as the use of thromboelastography, it is possible that administration of recombinant ATII might be beneficial if the target level of activity was maintained to provide an optimal heparin effect in the context of adequate clot strength. The evidence is scarce; however, 1 group reported the infusion of ATIII during ECMO, which led to decreased short-term bleeding complications. ${ }^{11}$ Despite efforts to optimize the balance between bleeding or thrombosis, strokes occurred in 3 of our 4 patients. In 1 patient, the stroke was possibly related to an unintentional dislodgement of the circuit tubing by the patient's legs that occurred just before the manifestation of neurologic symptoms. Two other patients experienced hemorrhagic strokes that were possibly hemorrhagic conversions of embolic strokes. Although the right angle metal cannula in the LA approach has worked in adults with the Novalung with minimal thrombus buildup in the LA, ${ }^{5}$ in infants and children, this approach certainly places these patients at risk of developing a LA thrombus. An autopsy performed on 1 of the patients with a hemorrhagic stroke revealed a large clot burden within the cannula and within the LA adjacent and posterior to the metal right angle cannula. Anticoagulation had been intermittently withheld for the 10 days before death because of the stroke, thereby complicating the interpretation of the timing of the development of the large LA clot burden. In the same patient, however, the Berlin cannula was patent and without any clot burden. The other infant who had experienced a clinical stroke early in their treatment and had received full anticoagulation for $>50$ days before their death also had thrombus in the LA on autopsy. Finally, the 23-day-old patient had episodes of atrial dysrhythmias that required initiation of amiodarone therapy; conceivably, the large metal cannula contributed to irritation of the atrial tissue and resulted in the observed rhythm disturbances. These observations led us to adopt an alternative approach for the fourth patient in our series, such that a novel cannulation technique was adopted for the LA blood return. A 10-mm Gore-Tex graft was placed in the posterior right atrium through a small incision. The graft was anastomosed to the perimeter of a surgically created atrial septal defect to provide direct blood return from the circuit to the LA while avoiding a foreign body projecting into the LA (Figure 1). Although this patient was only supported for 5 days before LT, no thrombus was noted in the LA at transplantation. This new approach may be safer, with 5 $\mathrm{mm} \mathrm{Hg}$ less cannula resistance at $550 \mathrm{~mL} / \mathrm{min}$ flow and improved hemodynamics within the LA. The main 
advantage of the new cannulation technique was the decreased risk of thrombosis, allowing less aggressive anticoagulation and, hence, decreasing the risk of hemorrhage.

With the PA to LA connections of the oxygenator, atrial level shunts are not tolerated, because they can result in high Qp/Qs flows, with a cyclical flow pattern if a left to right atrial shunt is present. In the patient with the AV canal repair at the transition to the paracorporeal lung assist device, a small residual atrial level shunt was present that led to substantial problems for the infant. In a challenging clinical course, the patient underwent cardiac catheterization, which demonstrated a Qp/Qs of 4:1 with high left ventricular end-diastolic pressures owing to a slightly unbalanced AV canal with small left-sided structures and worsening renal function. After closure of this residual atrial level shunt, the patient began to improve clinically but ultimately died of multiorgan dysfunction. On the basis of this experience, we recommend that any atrial level shunts present in these patients should be closed during or before transitioning to a paracorporeal lung assist device. Additionally, patients with concomitant cardiac disease with left-sided dysfunction might not be ideal candidates for this type of support.

Proper guidelines for the use of this type of therapy will come with more patient experience. On the basis of our experience, patients with pulmonary hypertension at or greater than the systemic pressure are potential candidates for therapy, unless they have underlying complex congenital heart disease or myocardial dysfunction deemed to be irreversible or reversible within a long period (ie, cardiomyopathy). The main question that has concerned us has been the candidacy of patients with subsystemic pulmonary pressures combined with severe lung disease for this therapy. These patients depend on substantial cardiac output through the oxygenator to provide adequate oxygenation. Subsystemic PA pressure will facilitate unpredictable competing native pulmonary blood flow that could lead to marginal oxygenation. For older children, the limited availability of the Novalung could be a barrier to using this therapy. Other oxygenators on the market in the United States have a pressure decrease similar to that of the Novalung, with excellent gas transfer characteristics that can be used for this therapy.

Potential technological developments could improve the safety and breadth of this therapy. Decreasing the thrombogenicity of the oxygenator and entire circuit will be of paramount importance and could include improved hemodynamics of the cannulas and connectors and minimizing the circuit length and surface coatings of the oxygenator. ${ }^{12-14}$ More substantial developments such as microfluidic-based engineered lung devices ${ }^{15-18}$ have potential for hemodynamically normal blood flow, biologically active coatings, and, even, endothelialization to minimize the need for anticoagulation. Biologically based therapies such as decellularized lungs that have been recellularized ${ }^{19,20}$ might prove to be the ultimate paracorporeal technology.

\section{CONCLUSIONS}

Paracorporeal lung assist device support of neonates and young children has been demonstrated to be feasible in the present early patient series. Continued optimization of anticoagulation and additional refinement of cannulation strategies will hopefully make this therapy applicable to patients needing short- to medium-term lung support after respiratory decompensation.

We acknowledge the creativity and dedication of all our perfusionists: Michael S. Longo, BS, CCP, Steve C. Raithel, BS, CCP, Mark P. Shepard, BSN, RN, CCP, Mark J. Anderson, MS, CCP, and John Poe, CCP. Special thanks to Mary Mehegan, RN, BSN, CCRN, Deirdre J. Epstein, RN, BSN, and our cardiac anesthesia team and dedicated cardiac intensive care unit nursing staff, and Dr Allan Doctor, Chief of the Division of Pediatric Critical Care Medicine.

\section{References}

1. Gazit AZ, Sweet SC, Grady RM, Huddleston CB. First experience with a paracorporeal artificial lung in a small child with pulmonary hypertension. J Thorac Cardiovasc Surg. 2011;141:e48-50.

2. Puri V, Epstein D, Raithel SC, Gandhi SK, Sweet SC, Faro A, et al. Extracorporeal membrane oxygenation in pediatric lung transplantation. J Thorac Cardiovasc Surg. 2010;140:427-32.

3. Schmid C, Philipp A, Hilker M, Arlt M, Trabold B, Pfeiffer M, et al. Bridge to lung transplantation through a pulmonary artery to left atrial oxygenator circuit. Ann Thorac Surg. 2008;85:1202-5.

4. Strueber M, Hoeper MM, Fischer S, Cypel M, Warnecke G, Gottlieb J, et al. Bridge to thoracic organ transplantation in patients with pulmonary arterial hypertension using a pumpless lung assist device. Am J Transplant. 2009;9: 853-7.

5. de Perrot M, Granton JT, McRae K, Cypel M, Pierre A, Waddell TK, et al. Impact of extracorporeal life support on outcome in patients with idiopathic pulmonary arterial hypertension awaiting lung transplantation. J Heart Lung Transplant. 2011;30:997-1002

6. Fischer S, Simon AR, Welte T, Hoeper MM, Meyer A, Tessmann R, et al. Bridge to lung transplantation with the novel pumpless interventional lung assist device NovaLung. J Thorac Cardiovasc Surg. 2006;131:719-23.

7. Bein T, Zimmermann M, Philipp A, Ramming M, Sinner B, Schmid C, et al. Addition of acetylsalicylic acid to heparin for anticoagulation management during pumpless extracorporeal lung assist. ASAIO J. 2011;57:164-8.

8. Hoganson DM, Gazit AZ, Sweet SC, Grady RM, Huddleston CB, Eghtesady P. Neonatal paracorporeal lung assist device for respiratory failure. Ann Thorac Surg. 2013;95:692-4

9. Elizur A, Faro A, Huddleston CB, Gandhi SK, White D, Kuklinski CA, et al. Lung transplantation in infants and toddlers from 1990 to 2004 at St. Louis Children's Hospital. Am J Transplant. 2009;9:719-26.

10. Gandhi SK, Grady RM, Huddleston CB, Balzer DT, Canter CE. Beyond Berlin: heart transplantation in the "untransplantable" J Thorac Cardiovasc Surg. 2008; 136:529-31

11. Agati S, Ciccarello G, Salvo D, Turla G, Undar A, Mignosa C. Use of a novel anticoagulation strategy during ECMO in a pediatric population: single-center experience. ASAIO J. 2006;52:513-6.

12. Shiose A, Takaseya T, Kim HI, Kobayashi M, Horai T, Rao S, et al. In vivo evaluation of a new surfactant polymer coating mimicking the glycocalyx of endothelial cells. ASAIO J. 2011;57:395-8

13. Polk AA, Maul TM, McKeel DT, Snyder TA, Lehocky CA, Pitt B, et al. A biohybrid artificial lung prototype with active mixing of endothelialized microporous hollow fibers. Biotechnol Bioeng. 2010;106:490-500. 
14. Yang Z, Tu Q, Maitz MF, Zhou S, Wang J, Huang N. Direct thrombin inhibitor-bivalirudin functionalized plasma polymerized allylamine coating for improved biocompatibility of vascular devices. Biomaterials. 2012;33: 7959-71.

15. Hoganson DM, Anderson JL, Weinberg EF, Swart EJ, Orrick BK, Borenstein JT, et al. Branched vascular network architecture: a new approach to lung assist device technology. J Thorac Cardiovasc Surg. 2010;140:990-5.

16. Hoganson DM, Pryor HI II, Bassett EK, Spool ID, Vacanti JP. Lung assist device technology with physiologic blood flow developed on a tissue engineered scaffold platform. Lab Chip. 2011;11:700-7

17. Kniazeva T, Epshteyn AA, Hsiao JC, Kim ES, Kolachalama VB, Charest JL, et al. Performance and scaling effects in a multilayer microfluidic extracorporeal lung oxygenation device. Lab Chip. 2012;12:1686-95.

18. Wu WI, Rochow N, Chan E, Fusch G, Manan A, Nagpal D, et al. Lung assist device: development of microfluidic oxygenators for preterm infants with respiratory failure. Lab Chip. 2013;13:2641-50.

19. Ott HC, Clippinger B, Conrad C, Schuetz C, Pomerantseva I, Ikonomou L, et al. Regeneration and orthotopic transplantation of a bioartificial lung. Nat Med. 2010;16:927-33.

20. Petersen TH, Calle EA, Zhao L, Lee EJ, Gui L, Raredon MB, et al. Tissueengineered lungs for in vivo implantation. Science. 2010;329:538-41

\section{Discussion}

Dr Mark D. Rodefeld (Indianapolis, Ind). I would like to congratulate Dr Hoganson on a very good presentation, and I would also like to thank you for providing me the report in advance.

I congratulate the Washington University group for their work in a very challenging subset of patients and for leading the way in new therapies to treat them. In this report, Dr Hoganson has summarized their experience with the use of paracorporeal lung assist devices to support neonates and young children with primary respiratory failure who are awaiting LT. Clearly, the waiting period for a suitable lung donor can be long, and many patients will not survive this waiting period.

This study, which was essentially a feasibility study, with a total of 4 patients, has shown that it is possible to successfully bridge these patients to LT using a paracorporeal oxygenator alone. Patients can be extubated, and this has obvious implications with regard to quality of life.

The alternative support modality is ECMO, which has a limited span of its own and also complicated issues of its own. In patients with primary lung failure, ventricular or pump failure might not be a problem, and, therefore, an oxygenator alone might be a feasible therapy. There is no question that this is a challenging group of patients.

I have several questions.

You might have mentioned it; I did not see it in the report, but how many oxygenator changes were required in these patients?

Dr Hoganson. One of the patients required 2 oxygenator changes. We did that at the bedside just by providing some additional support and clamping the circuit, it can be done in $\leq 60$ seconds. One additional oxygenator change occurred in another patient, so 3 total. That was 1 of the real advantages with the Quadrox-being able to monitor the pre- and postoxygenator pressures. You can see sort of at the bottom of this chart that that is actually the transoxygenator gradient, and we were able to see whether that had increased. It is certainly a marker of potential thrombus buildup and that was an indication to change the oxygenator, so it was nice to have that marker for these patients.
Dr Rodefeld. Were the devices examined for clot burden after they were removed?

Dr Hoganson. You know, they were, and we did not see any. Also, even at the end of this particular patient's course when LA thrombus was involved in that pump exchange, we fully flushed out the thrombus. A few were present in the oxygenator, a few specks of thrombus, but not a significant burden by any stretch, despite withholding anticoagulation for the previous several days.

Dr Rodefeld. A significant number of strokes occurred in this series of 4 patients. Were any of the strokes temporally related to the oxygenator change?

Dr Hoganson. The stroke in the 23-month-old patient was related to a bedside event when he was extubated and actually kicked the cannula off the oxygenator and experienced a hemorrhagic event at the bedside. Thankfully that was immediately stopped. He was taken to the operating room, and the device was weaned that day. He had an embolic stroke found on imaging after that. It is certainly a question whether hypotension precipitated the clinical events of that stroke. That was an unusual occurrence.

The other 2 strokes we have certainly presumed were somehow related to LA thrombus that built up as a result of the metal tip projection into the LA.

Dr Rodefeld. So, you are more concerned about cannulation issues as being the source of stroke?

Dr Hoganson. Yes, I think that was our greatest concern about a potential etiology for the other 2 strokes.

Dr Rodefeld. It seemed as though all the patients required ECMO support, but they had had highly variable amounts of time from ECMO support to support by an oxygenator alone. What were the factors influencing the decision there?

Dr Hoganson. It was mostly related to our maturation of an understanding of when we might use this support. The first patient received ECMO for 17 days. Obviously, once it was conceived, it required time to get supplies in and the approval and such. The 1 patient was treated for 10 days; that was actually the second patient. We had conceived the idea before that patient began ECMO that perhaps we would need to use this because the presumed diagnosis was ACD because of an older sibling who had had it. Then, for the patient with AV canal right lung hypoplasia, we had often discussed potentially putting this patient, who was struggling, on this device. However, because it is a new device, we wanted to proceed only after the patient had met the indications for ECMO. We did not put many patients de novo on the support. This patient was emergently given ECMO at the bedside, and we just decided to take it down that day. It was really an evolution as we decided who might or might not be candidates.

Dr Rodefeld. Next question. Can you foresee the possibility of transitioning a patient directly to an oxygenator alone and not using the intervening ECMO?

Dr Hoganson. Yes, I think so. Since this series, we have discussed it for a couple patients. We have been struggling to potentially avoid that other procedure and the potential for complications. I think once we are more comfortable with which patients will be good candidates and who would be well supported, we will be willing to do that.

Dr Rodefeld. ECMO obviously has its own complications and problems that come with it, a morbidity that comes with it. Perhaps, if a better device were available, direct conversion to 
oxygenator therapy might be easier to envision, because you could potentially avoid some of the issues that come with ECMO alone.

Also, that transitions me to my last question, which is, I know you have done some basic work in this area. What would you foresee as the ideal oxygenator or paracorporeal lung device? In the best of all possible worlds, we would prefer to have a device that we could use that has been designed expressly for passive flow conditions. You have reported on use of a device that was designed for use in an ECMO circuit and not expressly designed for passive flow use.

Dr Hoganson. Right. Sometimes one is at the right place at the right time. Also, I have done a lot of work in this area, as you alluded, so for the past 7 years, we have been working on an engineered lung assist device for this purpose. Also, the approach we have been taking is an engineering approach to create hemodynamically perfect blood flow in an engineered vasculature. I think this, combined with using a biomaterial within the scaffold, we will be able to have both perfect blood flow and endothelialization and thus no longer require using high-dose anticoagulation and could use either low or no anticoagulation. I think that will afford us a number of potential clinical options.

Certainly, the patients we have talked about today with a paracorporeal lung assist device would be perfect candidates, in addition to treating premature infants with premature lung disease and umbilical cannulation directly at birth without intubating them, potentially allowing some lung growth. Also, for older adolescents or adults, I think potential will exist, just as others have described, for an implantable lung assist device that could be a bridge to LT or possibly a destination therapy after a left ventricular assist device-type of maturation. I think really focusing on physiologic blood flow and endothelialization in a lung device will provide good support for these patients.

Dr Shaf Keshavjee (Toronto, Ontario, Canada). I enjoyed your presentation. Obviously you have been treating a very challenging group of patients. In Toronto, we have treated about 15 of these patients with a PA-to-LA Novalung configuration. Granted most of them were adults, but we have used this in children, down to about $20 \mathrm{~kg}$ in weight. We have used a simple PA cannula and LA cannula, either in the superior pulmonary vein or the LA appendage, and we really have not had any stroke problems at all. We moved to extubation, waking up, and ambulating, just as you presented in the picture of 1 of our patients.

I wondered why you chose to use the trans-right atrial cannulation technique with the interposition graft, the Berlin heart cannula? That appears to complicate the situation. In the setting of a low-flow situation, this might be a setup for stroke complications.

Dr Hoganson. Yes, I think a number of factors could have contributed to why our reasonably small infants had issues that your older patients did not. Certainly, 1 of the questions we were unable to answer with these patients was how much blood flow goes through the oxygenator and how much goes through the native lungs. Also, echocardiography of the neonate showed reversal of pulmonary blood flow in the pulmonary vascular and a huge jet coming out of the cannula and a very small amount coming out of the pulmonary vein. I think the overall majority of the blood flow was coming through the oxygenator, and I think that just set up a flow pattern sort of behind the metal tip right angle cannula that led to some stasis, and I think that is the hemodynamic explanation. Also, I think it was a very small atrium.

Dr del Nido. If you put a flow probe on your circuits, you will measure what is going out across the circuit and, with a low-resistance membrane, you can adjust that and know exactly what is occurring.

Dr Hoganson. But the contribution of the native lungs we were unable to state. Thus, I think that is a hemodynamic explanation. Also, I think, certainly for 1 patient, they had intermittent holding of the anticoagulation for the 10 days before death, and perhaps that contributed to the LA thrombus in that patient. I think that was likely. The other patient had full anticoagulation for several weeks before the autopsy and so I do not think that was the case. Thus, I agree. I think we certainly thought it was going to be fine, on the basis of your work. Also, because we had some serious clinical issues, we decided to use a different approach.

Dr Keshavjee. The point is that what is going through the native pulmonary circulation in this patient at this point is essentially irrelevant. If the patients are hemodynamically stable and have flow going through the Novalung, that is what is keeping them alive and decompressing the right side. As the pulmonary circulation becomes better, such as in your patient who did get better and could be weaned off, the flow in the Novalung will decrease, so it balances itself.

Dr del Nido. Dr Keshavjee, and David for you, there is some experience in France in treating primary pulmonary hypertension, not in patients who are hypoxic, by creating a shunt between the PA and the descending aorta, with some very encouraging results. Can you comment on the potential role of this procedure. Obviously, if you have primary lung failure or lung problems, that is not an issue. However, for primary pulmonary hypertension, could you comment on the role of both these techniques.

Dr Keshavjee. The issue with primary pulmonary hypertension and the particular option we have been discussing, where you can go PA to LA, is that you correct the physiology with your device. You offload the right heart and provide a low vascular resistance route to pump across.

The problem with the aorta-to-PA shunt is that it does not fix the physiology for the lung. In those 4 or 5 cases in France, they did have good palliation. We tried it once in a child, and it did not work well. Within about 36 hours, we had to go back, undo the shunt, and put a Novalung in. We got that patient through, but it was pretty close. Thus, the PA to aorta shunt did not fix that particular challenging patient for us. However, we did discuss it extensively, the appeal being that if you are really worried about whether you can successfully bridge the child to LT, it might be better to not require a device. We have pushed, however, toward saying we will get a lung, and we have bridged children for $\leq 6$ months on the device-that is enough time to get a lung in North America. 